\title{
Senility, Loneliness and Solitude
}

\author{
Zühal GÜLER ${ }^{1}$ and Nuran GÜLER ${ }^{2 *}$ \\ ${ }^{1}$ AIBÜ Faculty of Arts and Sciences Department of Sociology, Turkey \\ ${ }^{2}$ Cumhuriyet University Faculty of Health Science, Division of Nursing, Turkey
}

*Corresponding author: Nuran GÜLER, Associate Professor, Cumhuriyet University Faculty of Health Science, Division of Nursing Department, 58140 Sivas, Turkey

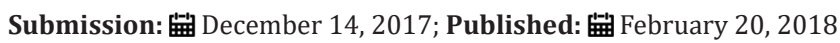

\begin{abstract}
When the relevant literature is examined, it can be stated that the concepts of loneliness and solitude are generally used together. In this study, however, these two aforementioned concepts have been considered separately and distinctively. According to TÜIK 2016 Data, the elderly population ( 65 years old and over) was 6 million 495 thousand 239 people in 2015. The proportion of the elderly population to the total population has increased by $4.7 \%$ from 1940 to $2015.43 .8 \%$ of the elderly population are males and $56.2 \%$ are females.

In addition to the rapid aging of Turkish people, another important feature that should be underlined is that the elderly people in Turkey live predominantly in rural areas. The loneliness and solitude of elderly people in both rural and urban areas are also increasing proportionally due to the migration from rural to urban areas, change in family structure, and etc. There is a also significant difference between the genders in terms of loneliness. As a matter of fact, according to TÜIK 2015 Data, in 2015, 76.5\% of the elderly people "living alone" are women and 23.5\% are men.

The theme of this study is to reveal the loneliness and solitude in senility on the axis of the rural and urban. The aim of the study is to examine the factors that contribute to the loneliness and solitude of elders such as age, gender, marital status, education, and poverty in the context of causal analysis. In the study, data collection via literature review technique, in other words secondary sources related to the subject will be utilized. In addition, the data of the project executed by Assist. Assoc. Dr. Zuhal Guler together with Assoc. Dr. Nuran Güler and Asst. Assoc. Dr. Doğancan Özsel titled "Residents in Nursing Homes: Senility in Rural Areas" and supported by TUBITAK will be used.
\end{abstract}

Keywords: Senility and ageing; Loneliness and solitude; Migration from rural to urban

\section{Introduction}

When the relevant literature is examined, it can be stated that the concepts of loneliness and solitude are generally used together. In this study, however, these two aforementioned concepts have been considered separately and distinctively.

According to TÜİK 2016 Data, the elderly population (65 years old and over) was 6 million 495 thousand 239 people in 2015 [1]. When the elderly population ratio is analyzed annually, the proportion of the elderly population to the total population was $3.5 \%$ in 1940 and in 1960, 4.7\% in 1980, 5.7\% in 2000 and $8.2 \%$ in 2010 and in 2015 . Hence, the proportion of the elderly population to the total population increased by $4.7 \%$ from 1940 to $2015.43 .8 \%$ of the elderly population are males and $56.2 \%$ are females. [1].

In addition to the rapid aging of Turkish people, another important feature that should be underlined is that the elderly people in Turkey live predominantly in rural areas. The loneliness and solitude of elderly people in both rural and urban areas are also increasing proportionally due to the migration from rural to urban areas, change in family structure, and etc. There is also significant difference between the genders in terms of loneliness. As a matter of fact, according to TÜİK 2015 Data, in 2015, $76.5 \%$ of the elderly people "living alone" are women and $23.5 \%$ are men [2]. The theme of this study is to reveal the loneliness and solitude in senility on the axis of the rural and urban. The aim of the study is to examine the factors that contribute to the loneliness and solitude of elders such as age, gender, marital status, education, and poverty in the context of causal analysis.

In this study, data collection via literature review technique, in other words secondary sources related to the subject will be utilized. In addition, the data of the project executed by Assist. Assoc. Dr. Zuhal Guler together with Assoc. Dr. Nuran Güler and Asst. Assoc. Dr. Doğancan Özsel titled "Residents in Nursing Homes: Senility in Rural Areas" and supported by TUBITAK will be used [3].

We principally give the definition of elderly people, explain the concepts of loneliness and solitude and the differences between them by taking into account the studies existing in the literature. The study is completed by assessment and conclusion after mentioning the reasons behind the loneliness of elders and in senility the loneliness and solitude in Turkey. 


\section{Senility, Loneliness and Solitude}

\section{Who is called as elderly?}

Who is the called as elderly? To be able to give a definite answer to this question is very difficult due to the nature of the concept of senility. Because just as in the case of gender and social gender differentiation, one can speak of age and social age. Senility is a relative phenomenon. It differs from community to community, culture to culture even epoch to epoch. In every society or in every culture who do we call as elderly changes as well as the meaning of senility and the roles of and expectations from the elderly people. From this point of view, it can be stated that "age" is a social category in sociological terms [4].

The World Health Organization (WHO) has a starting age of 65 for senility and accepts the old age as "over 65 years old". The use of different age criteria is also a question. However, there are differences between the institutions and foundations regarding the age at which senility starts. For example, whereas the studies in United Nations consider 60 years old and above in describing elderly people, in most of the national and international studies the definition of WHO is followed [5]. The elderly population is divided into subgroups such that the 65-74 age group is defined as "youngelderly", the 74-84 age group is defined as "elderly", the 85 and over age group as "old-elderly" [5]. However, it is possible to state that as the average life span gets longer, the starting age of senility may shift to older ages.

\section{Loneliness and solitude}

Generally, loneliness and solitude (being alone) are used interchangeably or together. However, these two phenomena are totally different from. That is, one can live alone, but not feel lonely, or without being alone, may feel lonely. "Loneliness refers to a subjective situation (the relationships of a person with other people), while solitude or being alone expresses an objective situation (with whom and with how many people the person is associated) [6].

"Loneliness", which is peculiar to mankind, is a phenomenon that has a social meaning like "age" does. Because the perception, meaning and interpretation of loneliness also differ according to societies, cultures, time, and even people. For this reason, it is like a pendulum with a two ends oscillating between positive and negative.

On one side of loneliness there are negative feelings such as "sadness, deprivation, abandonment, exclusion, alienation or un supportiveness, on the other side there are positive emotions like to be on his/her own, to be able to stand on his/her own, to be able support himself/herself, to be able to produce and to be productive and creative by" [7].

For example, according to Alptekin, "while Western cultures attribute more positive meaning to loneliness like individuation, separation, independence and self-sufficiency, in Eastern cultures and Mediterranean societies, it is accompanied by more negative connotations" [7]. In this sense, the policies of Western cultures for elderly people are intended to strengthen them by protecting their individuality and to ensure their living unaided. In our culture, on the other hand, the situation is exactly the opposite such that social support networks, especially family ties, are being tried to be strengthened and the care of the elderly is expected to be undertaken by the family members. "Man" is existentially alone in Cosmos, and as Bedri Rahmi Eyüboğlu also points out in his books, "you are only as much as your loneliness".

\section{Reasons of Loneliness and Solitude in Senility}

As mentioned before, philosophical sense of loneliness is specific to the human species, but the causes, consequences, and effects of loneliness, observed all along the line and in every age group, may vary according to the gender, marital status, health, age categories in senility. For this reason, the phenomenon of loneliness, which may sometimes extend to suicide in senility, should be considered as one of the concepts to be emphasized.

Numerous studies have been conducted on the causes of loneliness. Among these studies, the causes of loneliness in old age can be stated as follows: [1]

\section{Socio-demographic causes, physical and psycho-social causes, reasons caused by personality, reasons caused by insufficiency of social networks, cultural causes}

The feeling of loneliness of the elderly people varies according to the gender, marital status, the place where they accommodate an educational level. In literature there are different results indicating that elderly women or men feel more lonely. While in some studies, it is found that gender difference does not make a difference in loneliness in advanced adulthood [8,9], others indicate that, women are more lonely than men [10] and men are more lonely than women [11]. Although a variety of studies have shown different results in terms of gender, the elderly people feel lonely in different fields. According to this, "women feel themselves alone in terms of interpersonal relations, emotional and social self-efficacy perceptions, while men feel lonely in terms of perceived financial, instrumental and physical self-efficacy" [12]. Undoubtedly, this difference can be thought to be the effect of social gender perception.

Apart from gender, based on the results obtained in the related studies, other parameters affecting loneliness in advancing years and the relationship between senility and loneliness can be summarized as follows [6]:

A. As the age progresses, especially after the age of 80 , the loneliness increases.

B. The level of income, in other words, the absolute and relative poverty increase the loneliness in elders.

C. Elderly people whose spouses are dead, who have never got married and got divorced feel more lonely compared to the older people living with their spouses.

D. As the level of education decreases, the loneliness of the elders increases. 
E. Elderly people who do not have children feel more lonely than the ones who have children.

F. The elderly people living in rural areas feel more lonely compared to the elders living in urban areas.

G. Older people who are not physically and mentally healthy, feel more lonely than the old ones who are healthy.

H. The elderly people with low anger and tolerance threshold and with high level of stress, anxiety and fear are more lonely.

I. Older people with limited friendship associations who do not have sufficient emotional support in terms of social networking are more lonely.

According to Kapıkıran \& Kapıkıran [6], in countries with better health and economic assurance the elderly people to feel less lonely. Nevertheless, according to many northern European countries, loneliness can be experienced even though the level of prosperity is high. It can be thought that this is the effect of extreme individualization in the society.

\section{Reasons of Loneliness and Solitude in Senility in Turkey}

Turkish people have been aging rapidly and Turkey is not well prepared for this period in terms of infrastructure. While the aging of developed Western countries last 100 years, in Turkey it takes a short period of time around 30-40 years. The elderly population in Turkey has increased by $17.1 \%$ in the last five years and reached $8.3 \%$ in 2016 [1]. In $2016,61.5 \%$ of the elderly population is in the $65-74$ age group, $30.2 \%$ are in the $75-84$ age group, and $8.2 \%$ are in the 85 and over age group. Hence, the proportion of the elderly population to the total population increased by $4.8 \%$ from 1940 to $2016.43 .8 \%$ of the elderly population is male and $56.2 \%$ is female.

Because of migration from rural to urban, change in family structure, etc., the proportion of elderly people living alone in rural and urban areas is also increasing proportionally. At this point, an important point to underline is that elderly people live predominantly in rural areas. According to the results of the year 2015, in Turkey the life expectancy at birth has been 78 years for the general population, 75.3 years for men and 80.7 years for women [2].

In general, women are living longer than men and the life expectancy difference at birth is 5.4 years. While the proportion of widowed elderly men is $12.7 \%$, the proportion of elderly women is $50.4 \% .76 .7 \%$ of elderly people living alone are women. The proportion of the poor elderly male population increased to $17.8 \%$ in 2015. The proportion of poor elderly women increased to 18.6 $\%$ in $2015[10]$.

According to TÜİK 2017 Data $40.2 \%$ of the elderly people want to live with their children. The proportion of elderly people who want to go to senior center is only $7.7 \%$, and the most important reason for their will is that $48.9 \%$ of them did not want to be a burden on their children. The main source of happiness of elders is found to be their families with $64.2 \%$. In Turkey, there are 5 thousand 232 elderly people aged over 100 years.
In 2015 , the proportion of primary school graduates is $43 \%$, the ratio of graduates of secondary school or equivalent degree is $5.2 \%$, the ratio of high school or equivalent degree graduate is $5.6 \%$ and the ratio of high school graduates is $5.4 \%$. Educational status of elderly population shows significant differences according to the gender. That is, at all levels of education, the proportion of older men is higher than that of older women [2].

\section{Conclusion}

It can be stated that elderly people in Turkey are becoming lonelier. Along with the immigration from rural to urban, which has gained momentum since 1950, young people have migrated to big cities, and the elderly people have begun to live in the villages during summer to winter or during summer in the villages and during winter in the cities. Within this period, they have started to become lonely in both villages and cities. Especially the ones living in the village during summer and in the city during winter remain in limbo between rural and urban.

The elderly living in squatter houses as a result of immigration, are now subject to the urban transformation, where squatters are replaced with apartment buildings, will face unraveling in solidarity and become lonelier. In the study conducted by Güler [3]. In the villages of Zara, the depression rates in elders who live in the village both during the summer and winter and who live in the village during summer and in the city during winter were above the mean value of Turkey. For example, $60 \%$ of elderly women experience definite depression and $12 \%$ have possible depression. The proportion of elderly women without depression is only $27.7 \%$. In the same study, those who declare the loneliness as the most important problem are very close to one another for the elders living in the village (9.9\%) and for the ones living in the village during summer but in the city during winter (9\%) [13].

In addition, more than half of the female elders (55.4\%) have sleeping problems. The proportion of elderly people who do not have sleeping problems is $44.6 \%$. According to the studies in the literature, depression is the most frequent problem among the psychological problems experienced in senility [13]. According to TÜİK data, while suicide rates between the ages of 15 to 34 are gradually decreasing, it is increasing in elders yearly [14]. While the suicide rate in the age group of over 65 (TÜİK, 2015) was 7.83\% in 2000 , it reached $9.5 \%$ in $2007,11 \%$ in 2010 and $11.7 \%$ in 2015 .

In many studies [15], the greatest fears of the elderly are determined as follows: as to have one foot in the grave and thus require care, loneliness and dying alone. These fears also increase the rate of depression and suicidal tendencies in the in advancing years. The policies should be enhanced in this direction towards the elderly people, acting on the fact that the family tends to evolve from the traditional family to the core family due to immigration from rural to urban, the development of mass media, etc. It should not be forgotten that in Turkey, unlike in other developed countries, the elderly people live mainly in the countryside or they remain in limbo between the urban and rural. One way of improving the standard of living of elderly people is to make sure that they age 
actively. In other words, they should be encouraged to participate in a variety of activities by letting them establish social relations without being isolated from the society. Therefore, living spaces in urban areas should be designed with the ageing in mind, and social activities should be arranged not only for young people but also for elders.

\section{References}

1. (2017) Turkish Statistical Institute. Elderly with statistic. News Bulletin, Number: 24664.

2. Turkish Statistical Institute (2015) Suicides According to Age Group and Sex

3. Güler Z, Güler N, Özsel D (2014) Nursing homes villagers: Aging in the Countryside. TÜBITAK SOBAG 1001 Project Unpublished Project Report, Ankara.

4. Görgün Baran A (2003) Sociology of exuberance, aging: Interdisciplinary approach, problems and solutions-1. pp. 35-57.

5. Tezcan S, Seçkiner P (2012) Demographic change in Turkey; elderly perspectives, Elderly health problems and solutions, Palme Publishing, Ankara.

6. Kapıkıran S, Kapıkıran Acun N (2016) Oldness and Loneliness, Aging: Interdisciplinary Approach, Problems and Solutions-2, Nobel Publications, Ankara. pp. 79-104.

7. Altekin S (2017) On Loneliness.
8. De jong Gierveld J (1987) Developing and Testing a Model of loneliness. J Pers Soc Psychol 53(2): 119-128.

9. Nicolaisen M, Thorsen K (2014) Who are Lonely? Loneliness in Older, Adults age Groups (18-21 years old), Using two Measures of Loneliness. The International Journal of Aging and Human Development 78(3): 229257.

10. Steptoe A, Shankar A, Demakakos P, Wardle J (2013) Social Isolation, Loneliness and All-Cause Mortality in Older Men and Women. Proc Natl Acad Sci U S A 110(15): 5797-5801.

11. Uslu E, Bulduk S, Keçeci A (2013) The Relationship Between Loneliness Levels and Perceived Social Support of Living Individuals in Düzce Alien Nursing Home, $7^{\text {th }}$ National Congress on Aging: Active and Healthy Aging, pp. 54-60.

12. Fry PS, Debats DL (2002) Self-efficacy beliefs as predictors of loneliness and psychological distress in older adults. Int J Aging Hum Dev 55(3): 233-269.

13. Elkin N (2016) Evaluation of depression fever and life satisfaction in elderly people who attend to a family health center. Mersin University Journal of Health Sciences 9(1): 9-21.

14. Duru G, Özdemir L (2009) Causes of elderly suicide and preventive applications. Hacettepe University Faculty of Health Sciences Nursing Journal, pp. 34-41.

15.Güler Z, Güler N, Özsel D (2016) Being Old Women in Rural Areas. International Social Research Journal 1(2): 86-100.

\section{Your subsequent submission with Crimson Publishers will attain the below benefits}

International License

For possible submissions Click Here

Submit Article 